\title{
Direct Visualization of Independent Ta Centers Supported on Two-Dimensional $\mathrm{TiO}_{2}$ nanosheets
}

\author{
Supporting Information
}

Zhenyu Bo ${ }^{1}$, Nicholas E. Thornburg², Lingxuan Peng1, Jose Julio Gutierrez Moreno ${ }^{3}$, Michael Nolan ${ }^{3}$, Laurence D. Marks ${ }^{1}$, Justin M. Notestein ${ }^{2 *}$

1. Department of Materials Science and Engineering, Northwestern University, Evanston, IL, 60208

2. Department of Chemical and Biological Engineering, Northwestern University, Evanston, IL, 60208

3. Tyndall National Institute, University College Cork, Lee Maltings, Dyke Parade, Cork, Ireland

* Corresponding author email: j-notestein@northwestern.edu

\section{Experimental}

General remarks: Unless otherwise noted, all materials in this study were synthesized using standard air-free Schlenk line and glove box techniques. Tantalum precursors $\left(\mathrm{TaCl}_{5}, \mathrm{Ta}(\mathrm{OEt})_{5}\right)$ were obtained at the highest possible purity from Strem Chemicals. pTert-butylcalix[4]arene ("calix") was received from Sigma Aldrich (95\%). All metal precursors were stored in an Ar glovebox and used as-received. Toluene was distilled over $\mathrm{CaH}_{2}$ and degasified using standard techniques. $\mathrm{TiO}_{2}$ supports (nanosheets ("nsTiO2") were dehydrated at $110^{\circ} \mathrm{C}$ under dynamic vacuum ( $<30 \mathrm{mTorr}$ ) for 5 hours prior to use in synthesis. Synthesis apparatuses and sample storage containers were wrapped in aluminum foil and stored in the dark to prevent exposure to ambient light.

Synthesis of $\mathrm{TiO}_{2}$ nanosheet support (sample: $n s \mathrm{TiO}_{2}$ ): $\mathrm{nsTiO}_{2}$ were synthesized using a previously published hydrothermal synthesis protocol.1, 2 Briefly, $2.8 \mathrm{~mL}$ of concentrated hydrofluoric acid (48 wt\% solution, >99\%, Macron) was added to $20 \mathrm{~mL}$ of titanium butoxide (97\%, Sigma-Aldrich) in the PTFE liner of an autoclave vessel and was allowed to stir for 30 minutes. [CAUTION: concentrated hydrofluoric acid is an extremely corrosive contact poison and should be handled and stored with extreme care.] The autoclave vessel was then sealed and placed in a $200^{\circ} \mathrm{C}$ oven for 24 hours. 
The resulting solids were washed and centrifuged 3 times in ethanol (200 proof, SigmaAldrich), then washed and centrifuged in ultra-pure water until the $\mathrm{pH}$ of the centrate reached approximately 5 . The solids were then washed and centrifuged 3 times in a solution of $0.1 \mathrm{M} \mathrm{NaOH}$, then washed and centrifuged again several times with 18.2 $\mathrm{M} \Omega \cdot \mathrm{cm}^{-1}$ water until the $\mathrm{pH}$ of the centrate was below 8 . Finally, the $\mathrm{nsTiO}_{2}$ solids were allowed to dry in air in a $60^{\circ} \mathrm{C}$ oven overnight.

Grafted materials from Ta-calix[4]arene ("Ta-calix") precursor (sample: Ta-Calix): Following protocols previously published by some of us,, $41.2 \mathrm{mmol} \mathrm{TaCl} 5$ was added to a $40 \mathrm{~mL}$ toluene suspension of $1.2 \mathrm{mmol} \mathrm{p}$-tert-butylcalix[4] arene inside of an $\mathrm{Ar}$ glovebox. The reaction flask was equipped with a magnetic stirbar, sealed, and transferred to a Schlenk line under $\mathrm{N}_{2}$ flow. A reflux condenser was attached, and the flask's contents were refluxed for $14 \mathrm{~h}$. The suspension was cooled to $80^{\circ} \mathrm{C}$, and discrete $5.0 \mathrm{~mL}$ volumes were syringe-transferred under $\mathrm{N}_{2}$ purge to flasks containing variable amounts of a dehydrated $\mathrm{TiO}_{2}$ support; the resulting solution concentrations were 0.10.5 Ta-calix complexes per $\mathrm{nm}^{2}$ support surface area (see main text). The suspension was refluxed for $24 \mathrm{~h}$, after which the solids were vacuum-filtered in air, washed with anhydrous toluene and dried under dynamic vacuum (<30 mTorr) for $>24$ hours. Some materials were then subjected to atomic layer deposition (ALD; see section below). Materials with and without additional ALD treatment were further treated with ozone at $110^{\circ} \mathrm{C}$ for $3 \mathrm{~h}$ at a ramp rate of $15^{\circ} \mathrm{C} \mathrm{min}-1$ prior to microscopy and catalysis.

Grafted materials from Ta(OEt) 5 precursor (sample: Ta-Et) : $0.15 \mathrm{mmol}$ Ta(OEt)5 was added to a $10 \mathrm{~mL}$ toluene suspension of variable amounts of $\mathrm{TiO}_{2}$ support inside of an Ar glovebox; synthesis. A magnetic stirbar was added to the flask, which was then sealed and transferred to a Schlenk line under $\mathrm{N}_{2}$ purge. A reflux condenser was attached, and the contents were refluxed for $20 \mathrm{~h}$. The solids were vacuum-filtered in air and washed with anhydrous toluene $(200 \mathrm{~mL})$ before drying under dynamic vacuum ( $<30$ mTorr) for $>24 \mathrm{~h}$. Some solids were then subjected to further ALD treatment (see section below). Materials with and without ALD post-treatment were subjected to ozone treatment at $110^{\circ} \mathrm{C}$ for $3 \mathrm{~h}$ at a ramp rate of $15^{\circ} \mathrm{C} \mathrm{min}-1$ prior to imaging and catalysis. 
Atomic layer deposition ( $A L D$ ) of $\mathrm{Al}_{2} \mathrm{O}_{3}$ overcoats (sample: Ta-ALD): Thin films of $\mathrm{Al}_{2} \mathrm{O}_{3}$ were grown using atomic layer deposition (ALD) reactions performed in a custom flow reactor with a stainless steel bed. ${ }^{5}$ The reactor operated at $100^{\circ} \mathrm{C}$ with pressure maintained between 1.0-2.0 Torr using ultra-high purity $\mathrm{N}_{2}$ carrier gas. Alternating doses of trimethylaluminum (TMA, 99.7\%, Sigma-Aldrich) and $18.2 \mathrm{M} \Omega \cdot \mathrm{cm}^{-1}$ water were used to create a series of self-limiting reactions. ${ }^{6}$ [CAUTION: TMA is pyrophoric and should be handled and stored with extreme care.] One reaction cycle of TMA/ $\mathrm{H}_{2} \mathrm{O}$ consisted of the following protocol: (a) dosing TMA for $300 \mathrm{~s}$; (b) purging TMA for $300 \mathrm{~s}$; (c) dosing $\mathrm{H}_{2} \mathrm{O}$ for 300 seconds; (d) purging $\mathrm{H}_{2} \mathrm{O}$ for $300 \mathrm{~s}$.

Physical Characterization: The regular TEM images were acquired using the JEOL JEM2100 FasTEM operated at $200 \mathrm{kV}$. The single Ta atom on $\mathrm{TiO}_{2}$ high-angle annular dark field (HAADF) images were acquired using the JEOL JEM-ARM200CF aberration corrected 200kV STEM/TEM. To prepare the samples for imaging, <5mg of samples were dispersed in the ethanol solvent $(\sim 3 \mathrm{ml})$ then sonicated to have a homogeneous mixture. Then a few drops of the mixture were drop casted onto a lacey carbon copper TEM grid and air-dried. The post-imaging analysis were completed using ImageJ software, the nearest neighbor distance (NND) was calculated using the Nnd plugin provided by Cooperative Computing Group at Center for Advanced Vehicular Systems at Mississippi State University. The X-ray diffraction pattern was acquired by the Rigaku Ultima PXRD instrument at $40 \mathrm{kV}$. The XPS analysis was completed using the Thermo Scientific ESCALAB 250Xi XPS instrument. The Raman spectrum was acquired using the Acton TriVista CRS confocal Raman spectroscopy equipped with a $514.5 \mathrm{~nm} 2018$ model gas laser in room temperature. The surface area of the nanosheets were determined using a Micrometrics ASAP 2010 with specific surface areas calculated using the Brunauer-Emmett-Teller (BET) method. Thermogravimetric analysis (TGA) was carried out using a TA Instruments Q500 under 90\%/10\% $\mathrm{O}_{2} / \mathrm{N}_{2}$. Mass loss between $300{ }^{\circ} \mathrm{C}$ and $800{ }^{\circ} \mathrm{C}$ is assigned to the combustion of the grafted calixarene template and used to calculate the template loading. Ta loading was determined using a Thermo iCap7600 ICP-OES with sample digestion in a mixture of HF and aqua regia. 


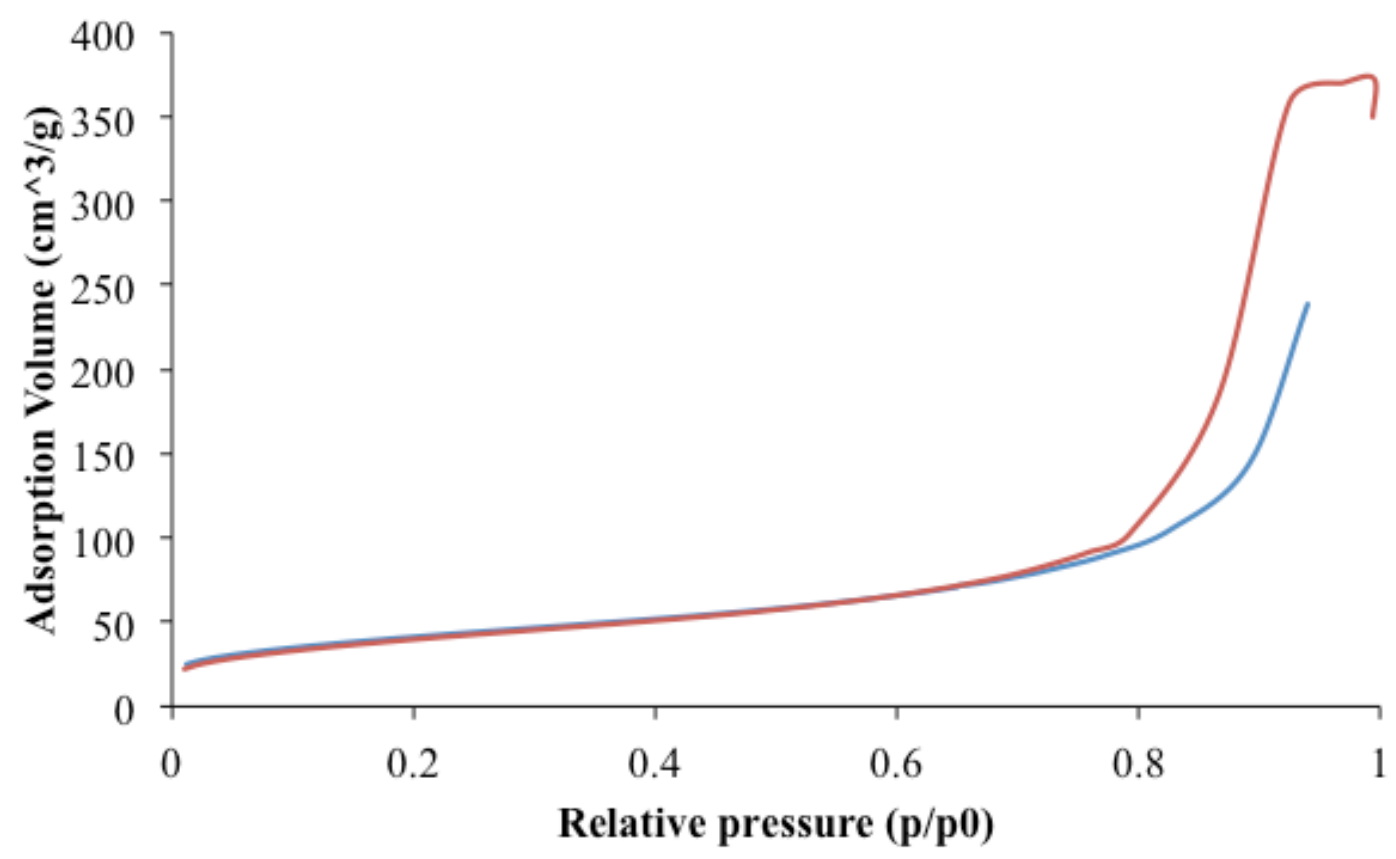

Figure S1. $\mathrm{N}_{2}$ adsorption/desorption isotherms of as-synthesized $\mathrm{nsTiO}_{2}$, corresponding to a BET surface area of $145 \mathrm{~m}^{2} / \mathrm{g}$.
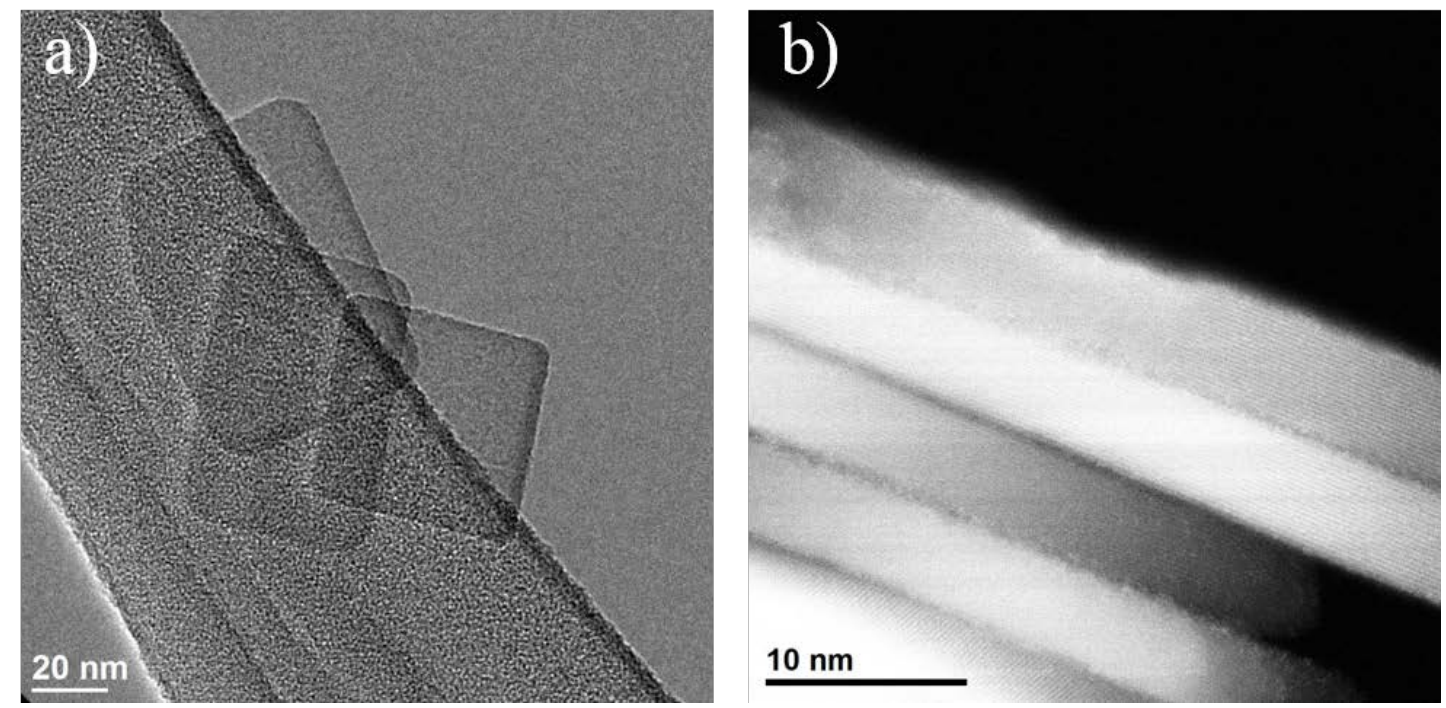

Figure S2. a) plain-view TEM image of $\mathrm{nsTiO}_{2}$ obtained using JEOL 2100 FasTEM. b) a stack of $\mathrm{nsTiO}_{2}$ imaged from the side showing the platelet thickness and minimal roughness. 


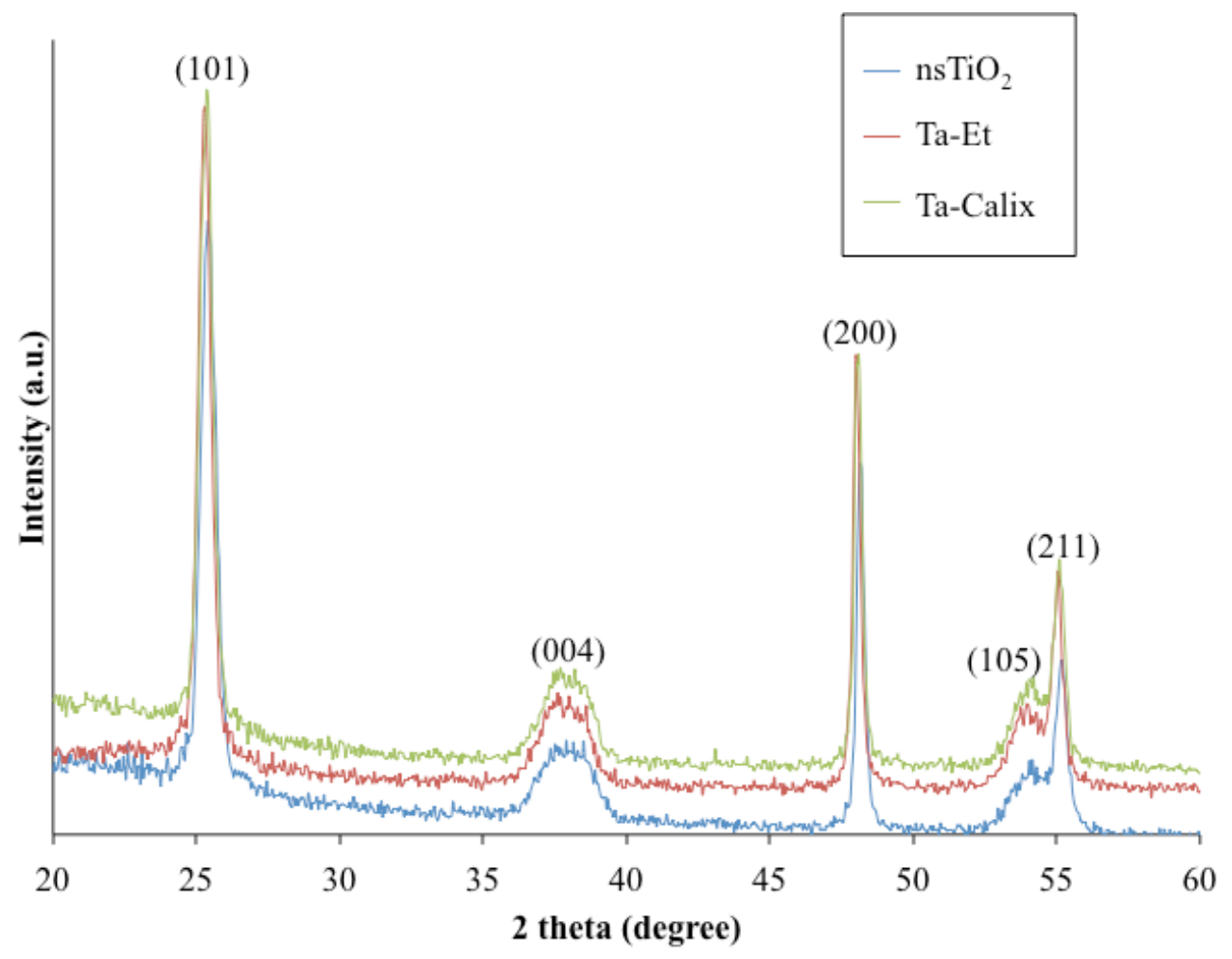

Figure S3. XRD patterns of $\mathrm{nsTiO}_{2}$, Ta-Calix, and Ta-Et showing only features corresponding to anatase $\mathrm{TiO}_{2}$ (labeled).

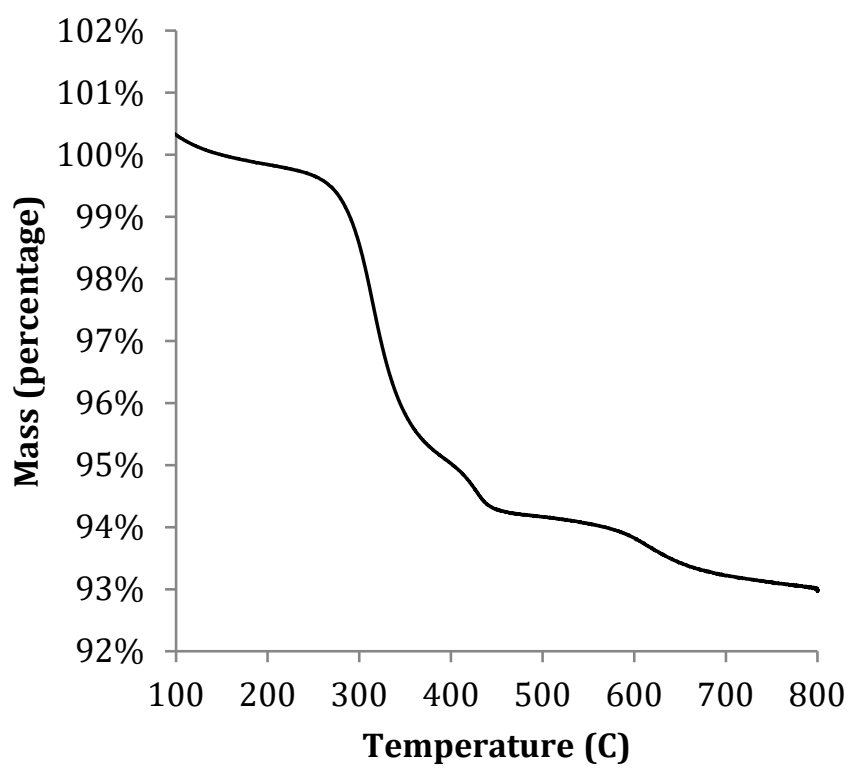

Figure S4. Thermogravimetric analysis of Ta-Calix in synthetic air showing combustion of the calixarene ligand as a mass loss near materials between $300^{\circ} \mathrm{C}$ and $450^{\circ} \mathrm{C}$. Mass loss is normalized to mass at $\sim 150^{\circ} \mathrm{C}$ to account for variable amounts of moisture or other volatiles on the sample surface. 

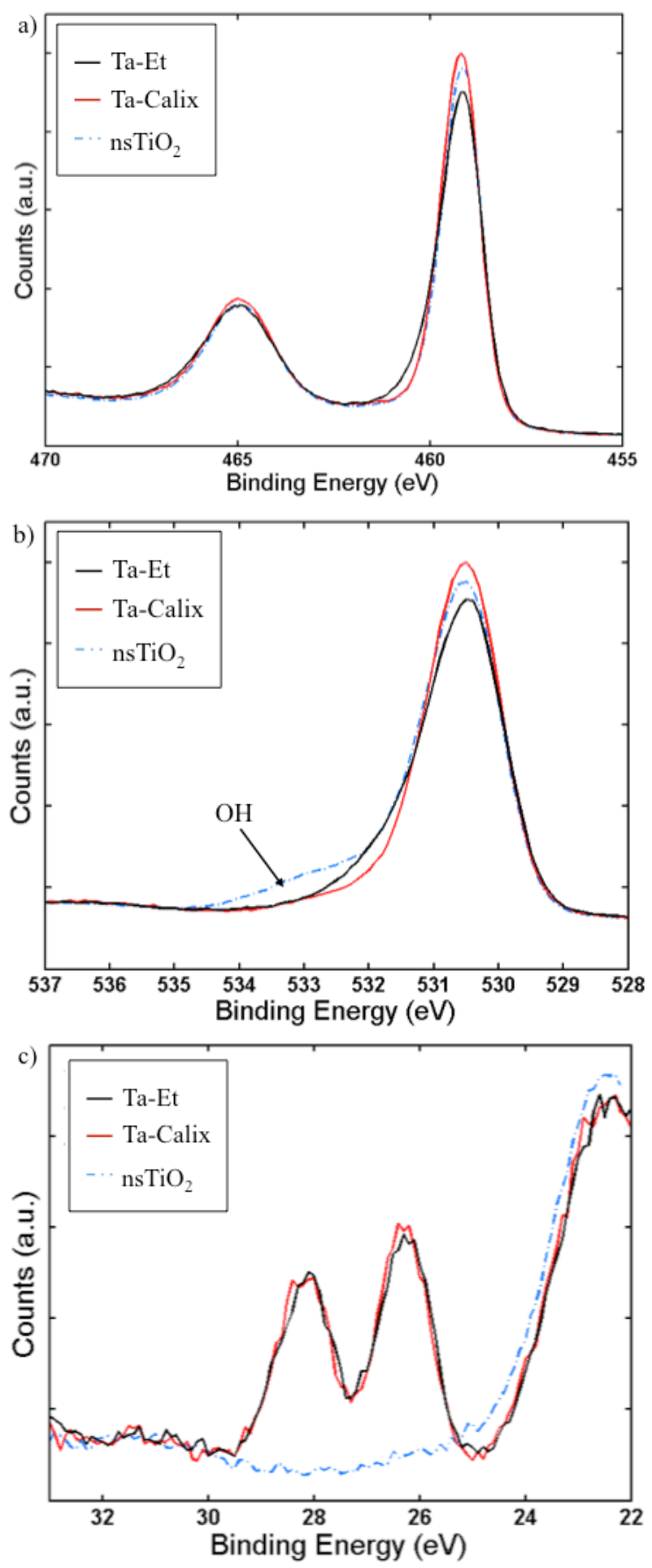

Figure S5. XPS of nsTiO 2 , Ta-Calix and Ta-Et a) Ti 2p, b) 0 1s, c) Ta 4f regions. The shoulder in the 01 s region of $\mathrm{nsTiO}_{2}$ is attributed to $\mathrm{OH}$ groups on the surface of $\mathrm{nsTiO}_{2}$. Peaks at $\sim 530 \mathrm{eV}$ and $\sim 22 \mathrm{eV}$ for all three samples are assignable to $\mathrm{O} 1 \mathrm{~s}$ and $\mathrm{O} 2 \mathrm{~s}$ in metal oxides. Otherwise, the spectra do not provide enough information to distinguish between TaCalix and Ta-Et. 


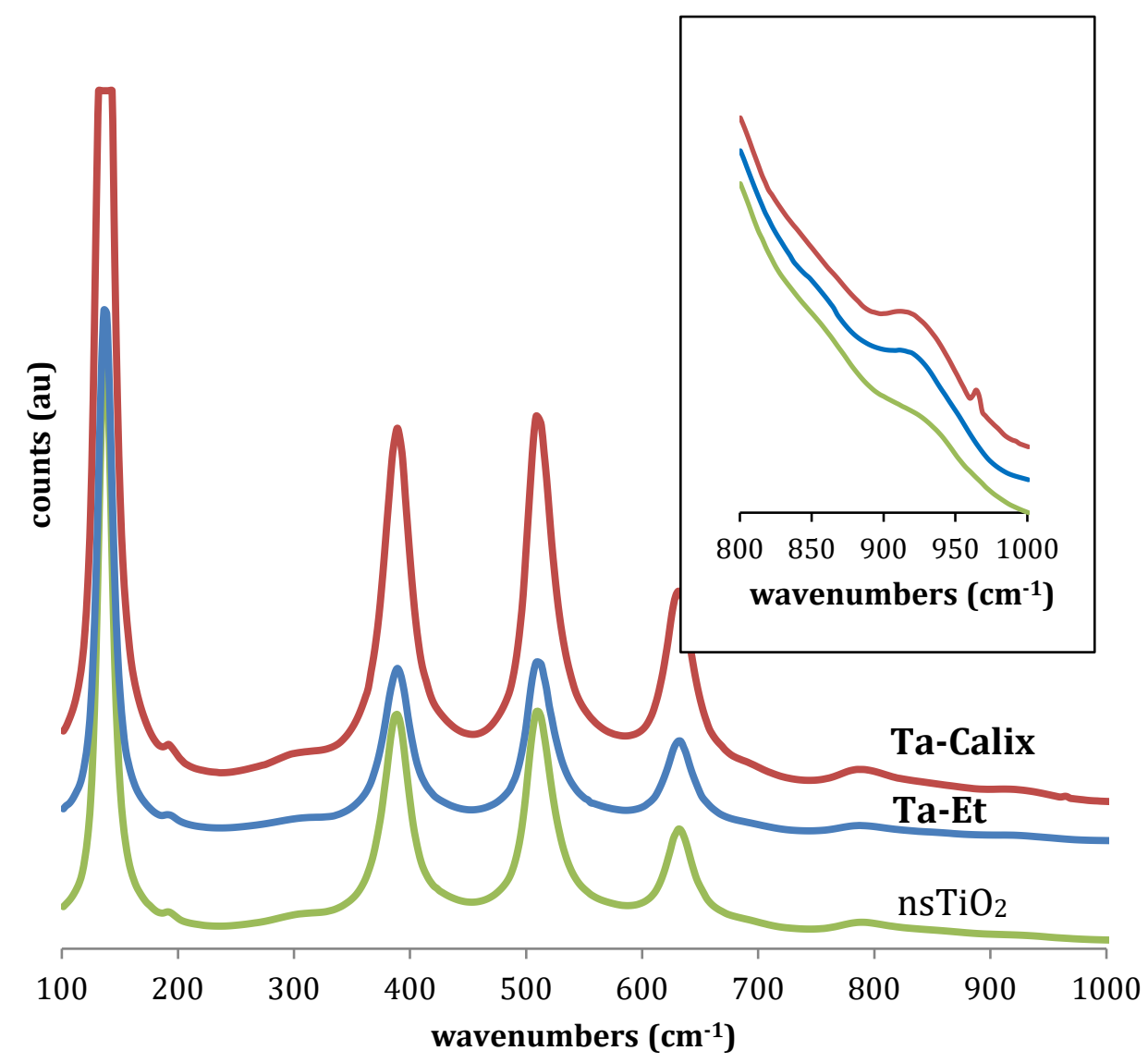

Figure S6. Raman spectra of $\mathrm{nsTiO}_{2}$, Ta-Et and Ta-Calix showing features corresponding to anatase $\mathrm{TiO}_{2}$. Inset shows Ta-associated peak near $925 \mathrm{~cm}^{-1}$. 


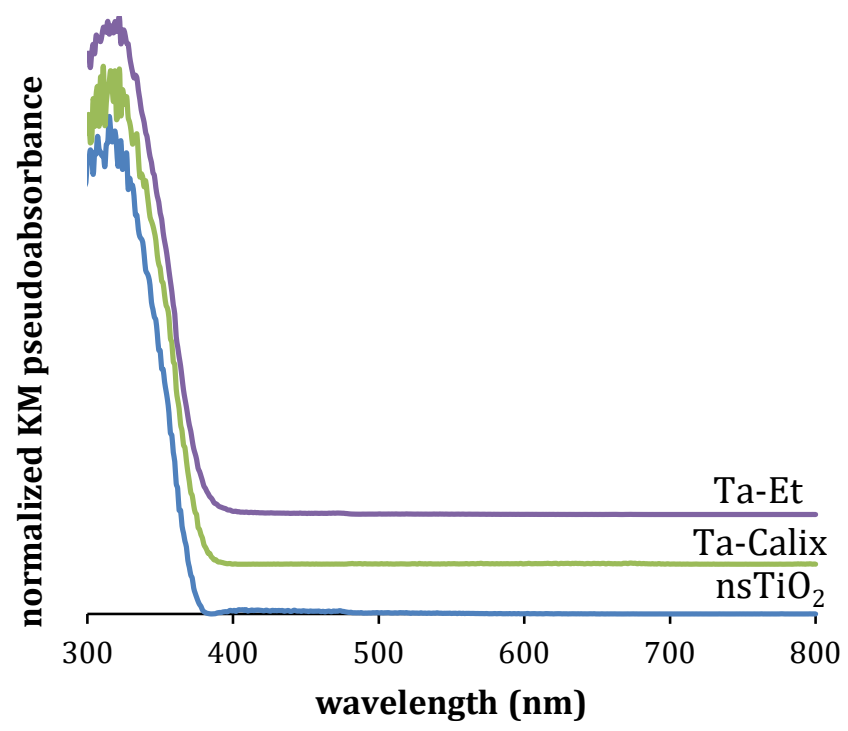

Figure S7. Normalized diffuse reflectance UV-visible spectra of samples Ta-Calix, Ta-Et and nsTiO2, which are essentially indistinguishable from one another.

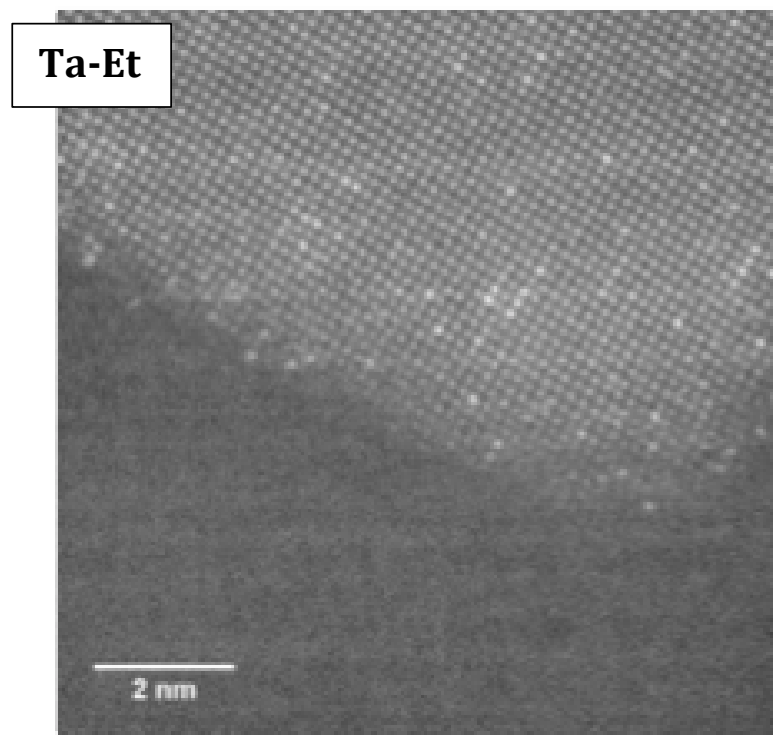

Figure S8. High Resolution TEM image of Ta-Et without median filter applied. 
a)

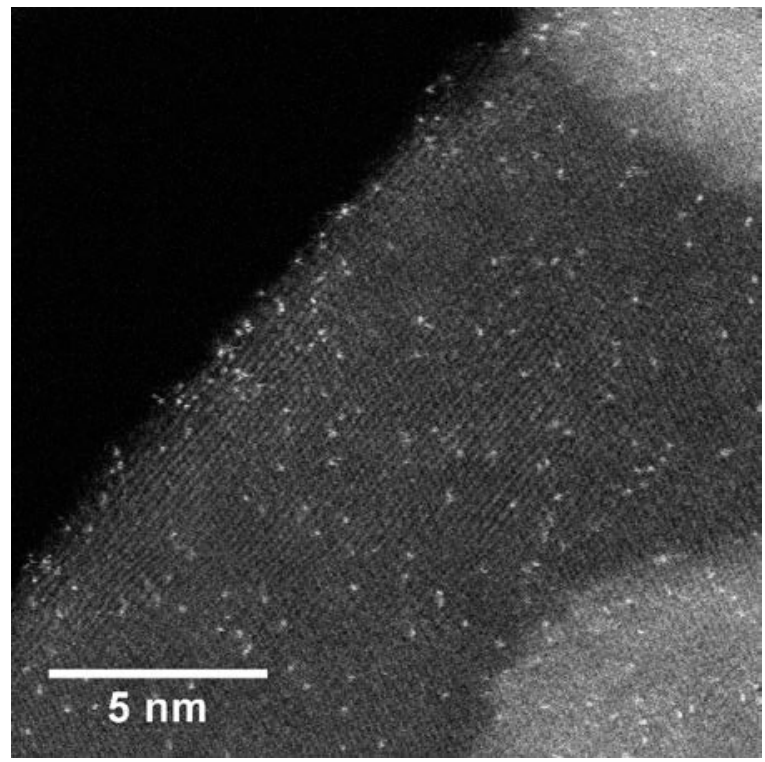

c)

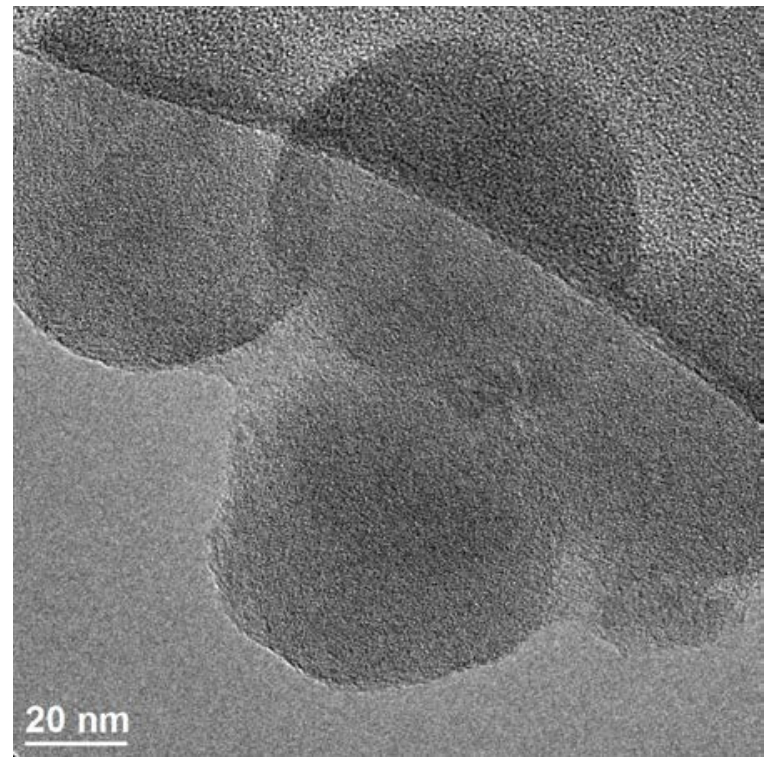

b)

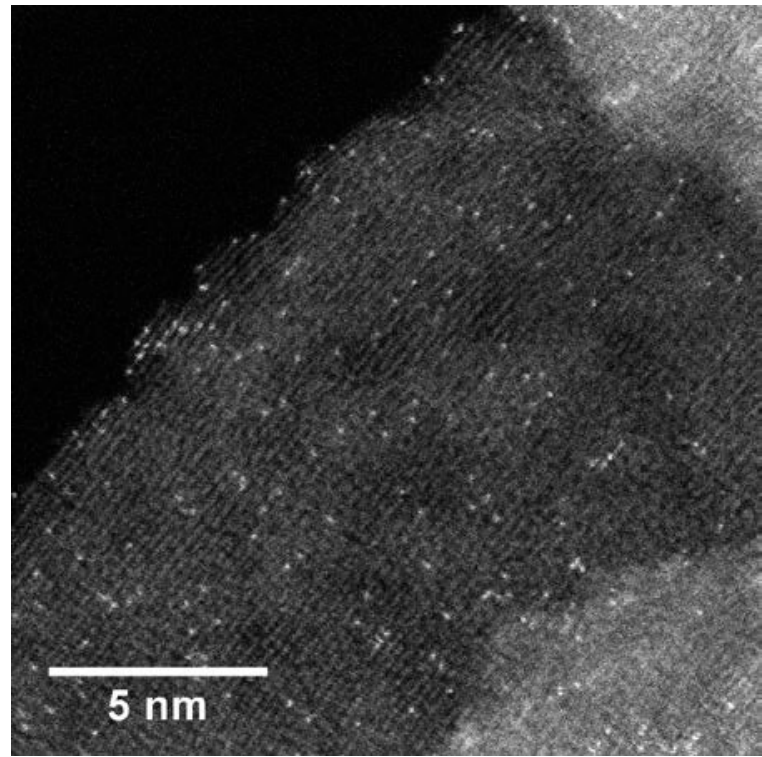

d)

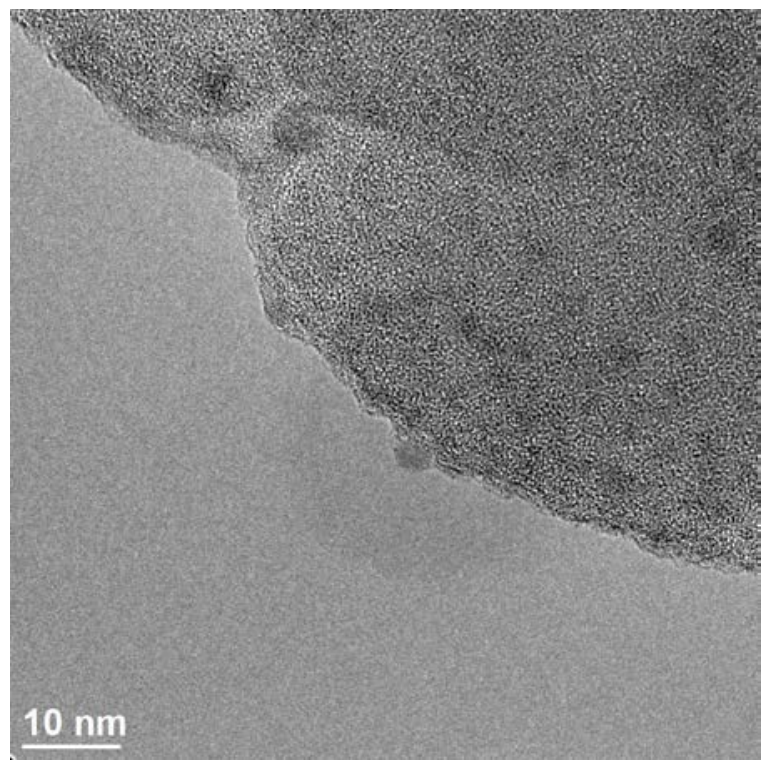

Figure S9. Sample Ta-Et initially (a) and after extended imaging exposure (b). Damage to the $\mathrm{nsTiO}_{2}$ is evident through the darkened sections and some roughening at the particle edge, but the Ta atoms do not aggregate to an appreciable extent. This is in contrast to a similarly-prepared $\mathrm{Ta}_{-} \mathrm{SiO}_{2}$ sample (c), in which the $\mathrm{Ta}$ obviously aggregates after extended exposure (d). 


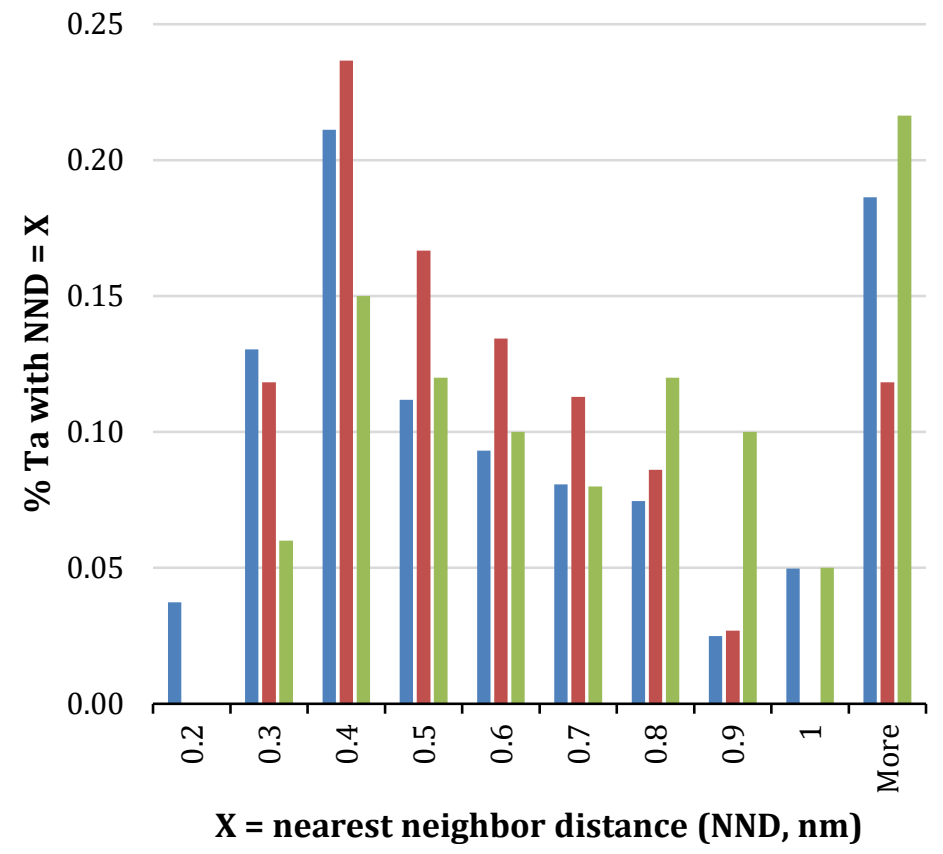

Figure S10. Histograms of nearest neighbor distances (NND) of Ta-Calix (green), Ta-Et (red) and c) Ta-ALD (blue) showing fewer Ta at distances $<0.4 \mathrm{~nm}$ for the Ta-Calix sample prepared from the bulky precursor than for Ta-Et. There is also evidence for some, but not extensive, rearrangement of the Ta positions after the ALD process. 


\section{COMPUTATIONAL DETAILS}

We perform Density Functional Theory (DFT) calculations within the framework of the Vienna Ab Initio Simulation Package (VASP 5.3.5)7. We use projector augmentedwave (PAW) potentials ${ }^{8,9}$ for the core-valence interaction while periodic plane waves with cut-off energy of $400 \mathrm{eV}$ describe the valence electrons in our system. The generalized gradient approximation given by Perdew and Wang (GGA-PW91) ${ }^{10}$ is used for the exchange correlation functional.

The (001) anatase $\mathrm{TiO}_{2}$ slab is 15 atomic layers thick, while a vacuum thickness of around $18 \AA$ avoid the interaction between the opposite sides of the surface though the 3-D periodic boundary conditions. To evaluate different coverage ratios, we built anatase $\mathrm{TiO}_{2}$ (001) 2x2 and 3x3 supercell expansion surfaces, keeping a constant thickness for the slabs. We found an anatase $\mathrm{TiO}_{2}$ equilibrium lattice parameter of $\mathrm{a}=$ $3.83 \AA$, thus our $2 \times 2$ supercell (containing 120 atoms) presents $\mathrm{a}=\mathrm{b}=7.66 \AA$ lattice parameters and the $3 \times 3$ supercell (containing 270 atoms) presents $a=b=11.50 \AA$ lattice parameters. A $(2 \times 2 \times 1)$ Monkhorst-Pack sampling grid was used for the simulations of the $2 \times 2$ supercell, while a $(1 \times 1 \times 1)$ Monkhorst-Pack grid is enough to reach energy convergence of the $3 \times 3$ surface. Gaussian smearing with $\sigma=0.05 \mathrm{eV}$ was used to integrate the Brillouin zone. The ionic relaxation and total energy convergence are assumed when all forces are $<0.02 \mathrm{eV} / \AA ̊$ and energy $<10^{-4} \mathrm{eV}$, respectively.

We perform DFT $+\mathrm{U}^{11}$ calculations to account for the presence of reduced $\mathrm{Ti}^{3+}$ species in $\mathrm{TiO}_{2}$ that may result from charge transfer between the adsorbed $\mathrm{Ta}$ and the anatase (001) surface. The DFT+U method defines a screened on-site Coulomb interaction, which help to localize the Ti $3 d$ electrons in our system. The Hubbard $+U$ parameter, applied on the Ti $3 \mathrm{~d}$ electrons, was set to $4.5 \mathrm{eV}$ which is widely used in previous DFT+U studies on $\mathrm{TiO}_{2}{ }^{12,13}$ while no correction is applied to Ta and $\mathrm{O}$ states. Bader charge analysis was then carried out with the software developed by Henkelman's group ${ }^{14}$.

We modify the $\mathrm{TiO}_{2}$ anatase (001) surface with $\mathrm{TaO}_{\mathrm{n}}$, where $\mathrm{n}=0-3$, and calculate the stability over a wide temperature range at $1 \mathrm{~atm}$ pressure, using the approach previously described in reference ${ }^{15}$. The thermodynamic formalism used is described in detail and applied in several studies. In brief, the (formal) formation of a tantalum oxide species on the titania surface from Ta metal and oxygen is:

$\left(\mathrm{TiO}_{2}\right)_{12}+\mathrm{Ta}+\frac{n}{2} \mathrm{O}_{2} \Leftrightarrow \mathrm{TaO}_{n} \bullet\left(\mathrm{TiO}_{2}\right)_{12}$ 
Where $\left(\mathrm{TiO}_{2}\right)_{12}$ denotes the stoichiometry of the surface supercell of $\mathrm{TiO}_{2}$ anatase (001). This reaction has the following change in surface free energy

$$
\gamma(T, p)=\frac{1}{A}\left[G\left[\mathrm{TaO}_{n} \bullet\left(\mathrm{TiO}_{2}\right)_{12}\right]-G\left[\left(\mathrm{TiO}_{2}\right)_{12}\right]-\mu[\mathrm{Ta}]-\frac{n}{2} \mu\left[\mathrm{O}_{2}\right](T, p)\right]
$$

Where $G\left[\mathrm{MO}_{n} \bullet\left(\mathrm{TiO}_{2}\right)_{12}\right]$ and $G\left[\left(\mathrm{TiO}_{2}\right)_{12}\right]$ are the Gibbs free energies of the supercells used to model $\mathrm{TaO}_{n}-\mathrm{TiO}_{2}$ anatase (001) and the $\mathrm{TiO}_{2}$ anatase (001) surface at the indicated stoichiometries. $\mu[\mathrm{Ta}]$ and $\mu\left[\mathrm{O}_{2}\right]$ are the chemical potentials of the solid $\mathrm{Ta}$ metal and oxygen. A is the surface area.

If we insert the following

$$
\Delta \mu[O](T, p)]=\frac{1}{2}\left[\mu\left[O_{2}\right](T, p)-E\left(O_{2}\right)\right]
$$

Into Eq. (1) above, we obtain

$$
\gamma(T, p)=\frac{1}{A}\left[G\left[\mathrm{TaO}_{n} \bullet\left(\mathrm{TiO}_{2}\right)_{12}\right]-G\left[\left(\mathrm{TiO}_{2}\right)_{12}\right]-\mu[\mathrm{Ta}]-\frac{n}{2} E\left[O_{2}\right]-n \Delta \mu[O](T, p)\right]
$$

The chemical potential of oxygen is related to the pressure and temperature conditions by assuming that the $\mathrm{TiO}_{2}$ surface is in thermodynamic equilibrium with the gas phase. Since the $\mathrm{O}_{2}$ atmosphere forms an ideal gas reservoir, the pressure dependence of $\Delta \mu[0](T, p)$ at a given temperature is given by $\left(\Delta \mu\left[\mathrm{O}_{2}\right](0 \mathrm{~K})=\mathrm{E}\left[\mathrm{O}_{2}\right]\right.$ and $p^{0}$ is the pressure of a reference state, $p=1 \mathrm{~atm})$.

$$
\Delta \mu[O](T, p)]=\frac{1}{2}\left[H\left(T, p^{0}\right)-H\left(0 K, p^{0}\right)-T S\left(T, p^{0}\right)+R T \ln \left(p / p^{0}\right)\right]
$$

In Eq. (4), the enthalpy, $H$, and entropy, $S$, at the temperature, $T$, can be taken from tabulated values [10]. $\mu[$ Ta] is set equal to the DFT total energy of the metallic bulk Ta, Ebulk[Ta].

The Gibbs free energies of the solid components in Eq. (3) are equated to total energies from DFT that are calculated in our fixed volume supercells and at $T=0 \mathrm{~K}$, so that:

$$
\gamma(T, p)=\frac{1}{A}\left[E\left[\mathrm{TaO}_{n} \bullet\left(\mathrm{TiO}_{2}\right)_{12}\right]-E\left[\left(\mathrm{TiO}_{2}\right)_{12}\right]-E_{\text {bulk }}[\mathrm{Ta}]-\frac{n}{2} E\left[O_{2}\right]-n \Delta \mu[O](T, p)\right]
$$

The vibrational contributions and $p V$ terms are neglected in this analysis. It has been previously shown that the vibrational contributions to the entropy tend to cancel out, and the influence of the $p V$ term is even smaller ${ }^{16-18 .}$

For a given chemical potential, we can then predict which $\mathrm{TaO}_{n} \bullet\left(\mathrm{TiO}_{2}\right)_{12}$ surface structure is the most stable by searching for the surface model with the lowest surface free energy. 


\section{COMPUTATIONAL RESULTS}

We perform Density Functional Theory corrected for on-site Coulomb interaction $(\mathrm{DFT}+\mathrm{U})^{11}$, with Hubbard $+\mathrm{U}$ parameter applied on the Ti $3 \mathrm{~d}$ electrons, to account for potential reduced $\mathrm{Ti}^{3+}$ species that may from due to charge transfer between the doping Ta and the $\mathrm{TiO}_{2}$ surface. Figure $\mathrm{S} .10$ shows the stability plot as a function of the temperature at 1 atm pressure for the different $\mathrm{TaO}_{n}(n=0-3)$ species deposited on $\mathrm{TiO}_{2}$ anatase (001) 3x3 supercell model surface along with their final relaxed atomic structures. The $2 \times 2$ supercell is shown in Figure S.11. We use Bader charge analysis to identify the oxidation states of the different Ti in our system. From Bader analysis, the $\mathrm{Ti}^{4+}$ species (grey spheres in Figure S.10) are characterized by Bader charges that lie between +2.72 and +2.64 electrons (from the difference between the Ti valance and the computed charge of 1.28 and 1.34 electrons). The $\mathrm{Ti}^{3+}$ species (green spheres) have Bader charges that lie between +2.3 and +2.24 electrons. Computed spin mangetisations on $\mathrm{Ti}^{4+}$ sites are $0 \mu$ в while they are $0.9-0.95 \mu_{\text {в }}$ on $\mathrm{Ti}^{3+}$ sites. The reduced $\mathrm{Ti}^{3+}$ species formed in the $\mathrm{TiO}_{2}$ surface due to charge transfers in the $\mathrm{Ta}-\mathrm{TiO}_{2}$ system progressively convert into $\mathrm{Ti}^{4+}$ upon $\mathrm{O}$ addition. The stability plot shows ' $\mathrm{TaO}_{3}$ ' - the model with 30 atoms added - as the most stable system, and no reduced $\mathrm{Ti}^{3+}$ were detected in this structure. The $\mathrm{TaO}_{\mathrm{n}}$ introduces a quite localized distortion in the $\mathrm{TiO}_{2}$ anatase lattice, as can be observed in the top view of our relaxed structures. The DFT relaxed structures show the most energetically favoured adsorption for Ta directly on top of the Ti-O columns, in line with the observed on the High-resolution HAADF-STEM images. 

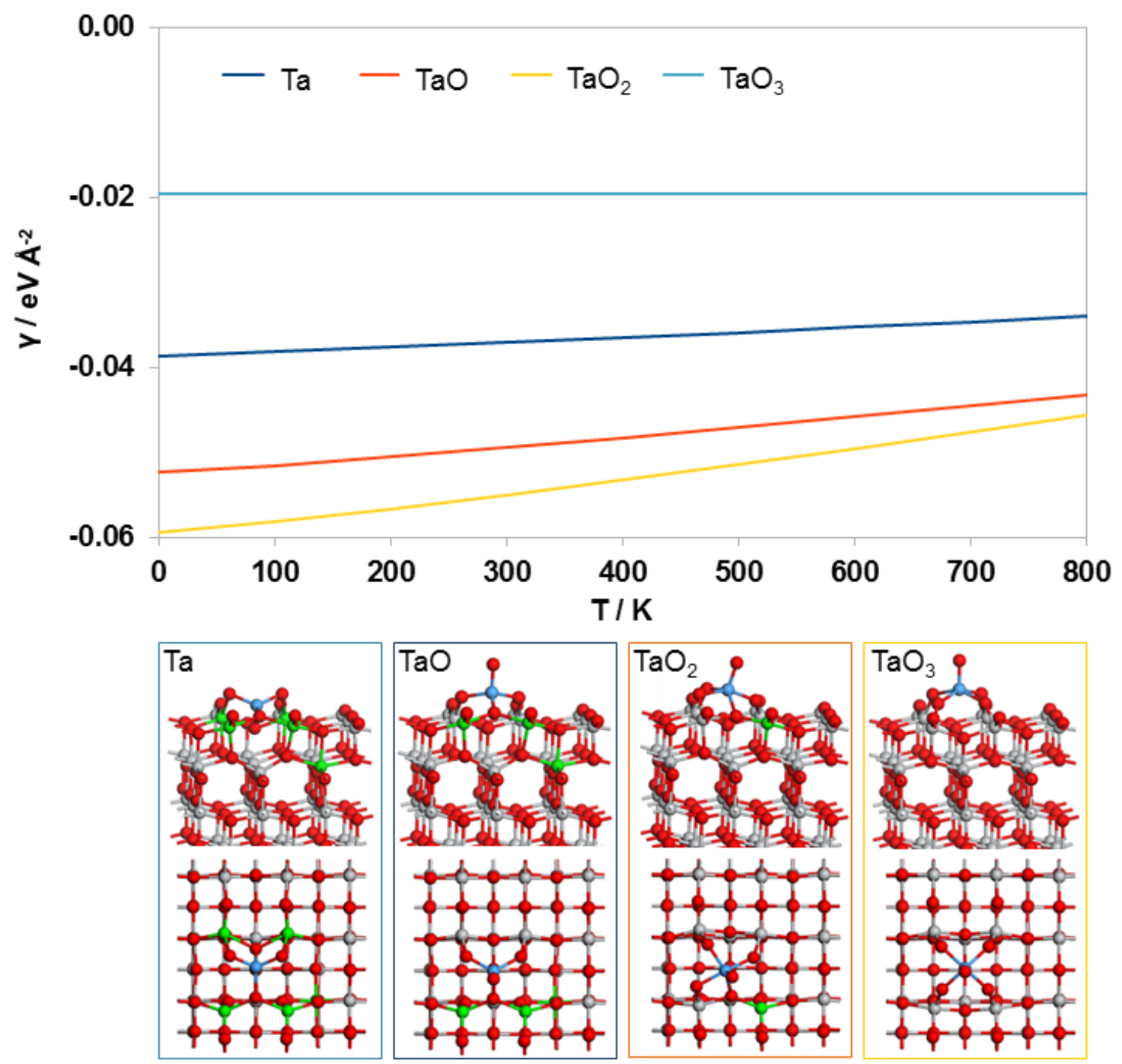

Figure S11. Surface free energy $(\gamma)$ of the $\mathrm{TaO}_{n}(\mathrm{n}=0-3)-\mathrm{TiO}_{2}$ system as a function of temperature at 1 atm pressure (top graph) and DFT relaxed structure of Ta supported on $\mathrm{TiO}_{2}$ anatase (001) surface (bottom boxes). The red balls stand for 0 atoms, the grey balls stand for $\mathrm{Ti}^{4+}$ atoms, the green balls stand for $\mathrm{Ti}^{3+}$ atoms and the blue ball represents a Ta atom. A colour code is used to identify the surface free energy trend lines in the energy plot with the surface model structures. 

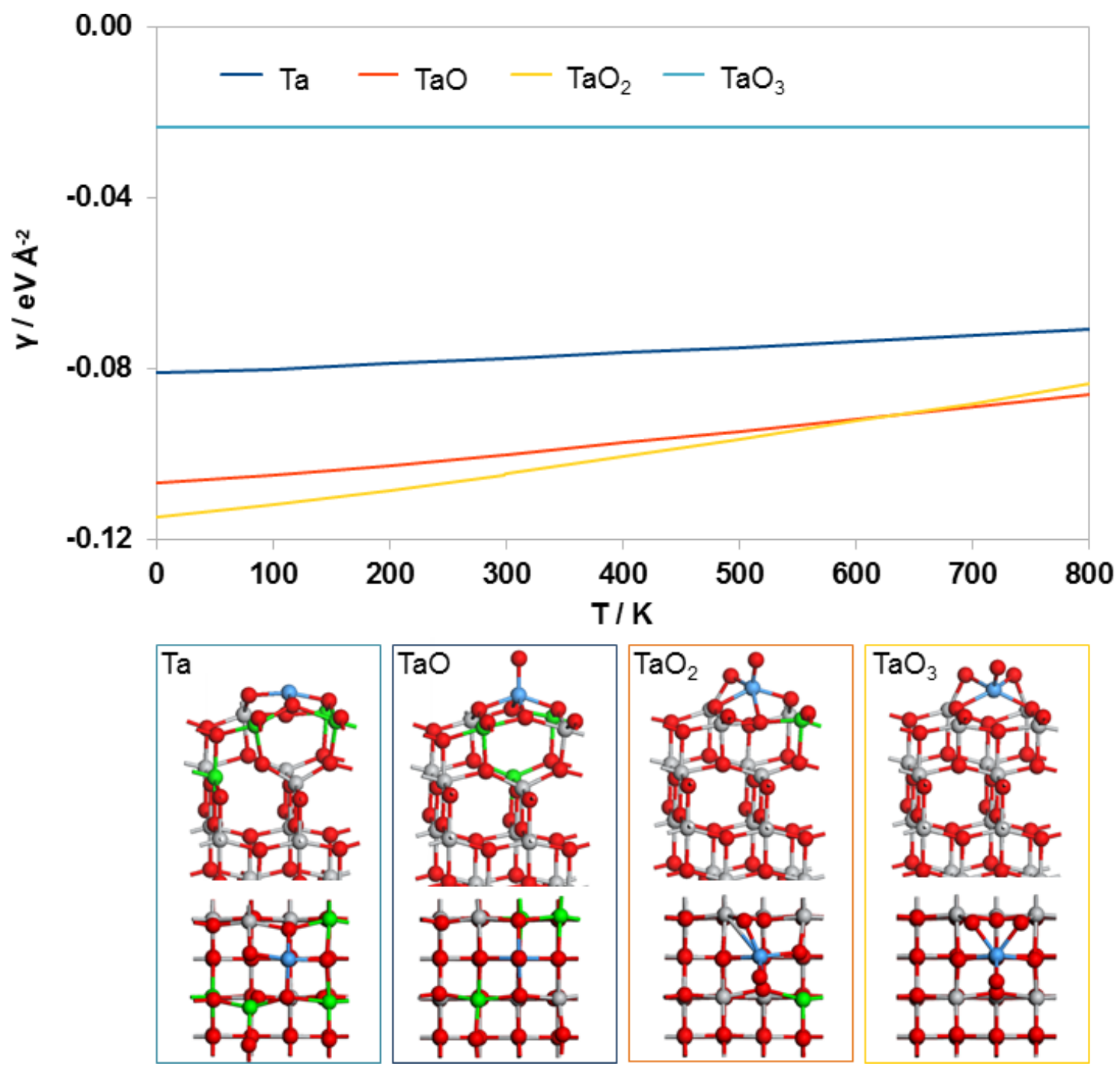

Figure S12. Surface free energy $(\gamma)$ of the $\mathrm{TaO}_{\mathrm{n}}(\mathrm{n}=0-3)-\mathrm{TiO}_{2}$ system as a function of temperature at $1 \mathrm{~atm}$ pressure (top graph) and DFT relaxed structure of Ta supported on a $\mathrm{TiO}_{2}$ anatase (001) $2 \times 2$ supercell model surface (bottom boxes). The red balls stand for $\mathrm{O}$ atoms, the grey balls stand for $\mathrm{Ti}^{4+}$ atoms, the green balls stand for $\mathrm{Ti}^{3+}$ atoms and the blue ball represents a Ta atom. A colour code is used to identify the surface free energy trend lines in the energy plot with the surface model structures. 

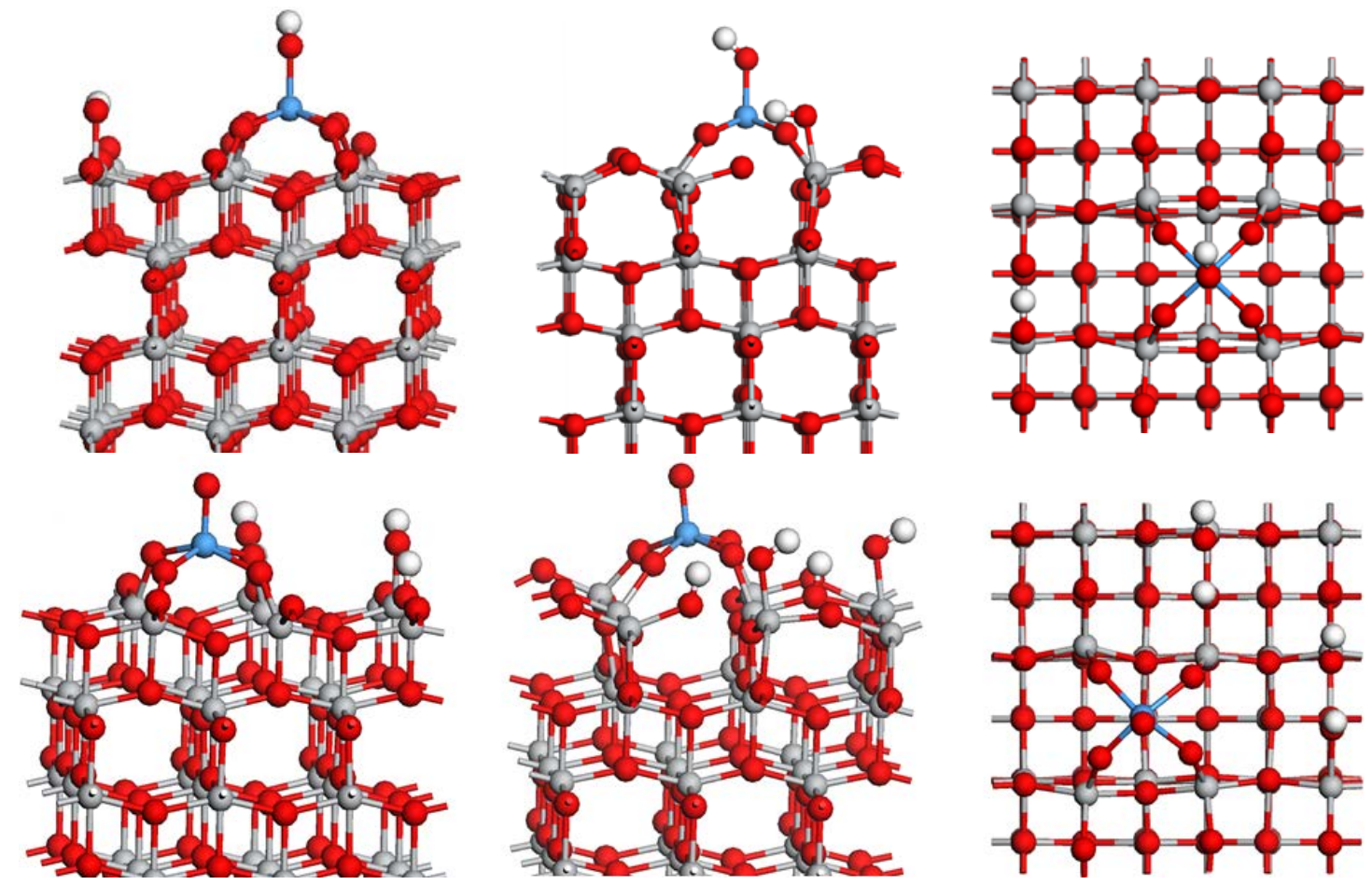

Figure S13. Additional DFT relaxed structures. Top) ' $\mathrm{TaO}_{3}$ ' on a $\mathrm{TiO}_{2}$ anatase (001) 3x3 supercell model surface with subsequent addition of $\mathrm{H}_{2} \mathrm{O}$ to generate one surface $\mathrm{TiOH}$ and one $\mathrm{TaOH}$. Bottom) ' $\mathrm{TaO}_{3}$ ' on a previously hydroxylated $\mathrm{TiO}_{2}$ anatase (001) 3x3 supercell model surface. From left to right: the front, side and top views are shown. The hydroxylated surfaces have been simulated by the dissociative adsorption of one $\mathrm{H}_{2} \mathrm{O}$ molecule on the ' $\mathrm{TaO}_{3}$ ' model of Figure $\mathrm{S} .10$ (top case) or two $\mathrm{H}_{2} \mathrm{O}$ molecules at the $\mathrm{TiO}_{2}$ surface. The red balls stand for $\mathrm{O}$ atoms, the white balls stand for $\mathrm{H}$ atoms, the grey balls stand for $\mathrm{Ti}^{4+}$ atoms and the blue ball represents a Ta atom. We do not observe reduced $\mathrm{Ti}^{3+}$ species in either system, and the $\mathrm{Ta}$ atom remains $\mathrm{Ta}^{5+}$. As in the above models and the experimental observations, the Ta atoms are located above the Ti-O columns. 

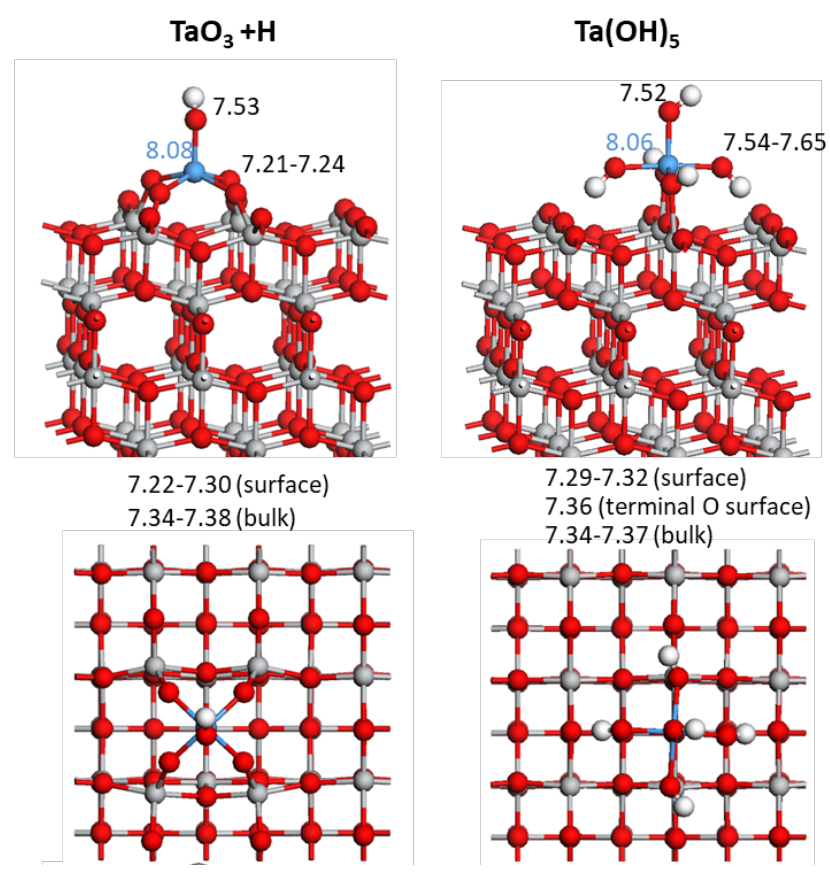

Figure S14. Side and top views of additional DFT relaxed structures. Left) ' $\mathrm{TaO}_{3}$ ' on a $\mathrm{TiO}_{2}$ anatase (001) $3 \times 3$ supercell model surface with subsequent addition of $\mathrm{H}$ to generate an apical TaOH. Right) The left model with dissociative addition of two $\mathrm{H}_{2} \mathrm{O}$ molecules to generate a $\mathrm{Ta}(\mathrm{OH})_{5}$ moiety on top of the $\mathrm{TiO}_{2}$ lattice. As in all models above, the $\mathrm{Ti}$ atoms remain $\mathrm{Ti}^{4+}$, and the $\mathrm{Ta}$ atom remains $\mathrm{Ta}^{5+}$ and is located above a $\mathrm{Ti}-\mathrm{O}$ column. Interestingly, the Ta atom moves from one lattice position to another as it becomes hydrated or dehydrated. This would not be distinguishable from the experimental TEM. 


\section{REFERENCES}

1. Han, X.; Kuang, Q.; Jin, M.; Xie, Z.; Zheng, L. J. Am. Chem. Soc. 2009, 131, 3152-3153.

2. Liang, Y. T.; Vijayan, B. K.; Lyandres, O.; Gray, K. A.; Hersam, M. C. J. Phys. Chem. Lett. 2012, 1760-1765.

3. Morlanés, N.; Notestein, J. M. J. Catal. 2010, 275, 191-201.

4. Thornburg, N. E.; Thompson, A. B.; Notestein, J. M. ACS Catal. 2015, 5, 5077-5088.

5. Elam, J. W.; Groner, M. D.; George, S. M. Rev. Sci. Instrum. 2002, 73, 2981-2987.

6. Groner, M. D.; Fabreguette, F. H.; Elam, J. W.; George, S. M. Chem. Mater. 2004, 16, 639-645.

7. Hafner, J. J. Compu. Chem. 2008, 29, 2044-2078.

8. Blochl, P. E. Phys Rev B 1994, 50, 17953-17979.

9. Kresse, G.; Joubert, D. Phys Rev. B 1999, 59, 1758-1775.

10. Perdew, J. P.; Wang, Y. Phys. Rev. B 1992, 45, 13244-13249.

11. Liechtenstein, A. I.; Anisimov, V. I.; Zaanen, J. Phys. Rev. B 1995, 52, R5467-R5470.

12. Iwaszuk, A.; Nolan, M. Phys. Chem. Chem. Phys. 2011, 13, 4963-4973.

13. Graciani, J.; Plata, J. J.; Sanz, J. F.; Liu, P.; Rodriguez, J. A. J. Chem. Phys. 2010, 132, 104703.

14. Tang, W.; Sanville, E.; Henkelman, G. J. Phys. Condens. Matter 2009, 21, 084204.

15. Popa, C.; Ganduglia-Pirovano, M. V.; Sauer, J. J. Phys. Chem. C 2011, 115, 7399-7410.

16. Stull, D. R.; Prophet, H. JANAF thermochemical tables; DTIC Document: 1971.

17. Hofmann, A.; Sauer, J. J. Phys. Chem. B 2004, 108, 14652-14662.

18. Reuter, K.; Scheffler, M. Phys. Rev. B 2001, 65, 035406. 